\title{
Escudo de Blumer: signo diagnóstico de la diseminación a distancia del cáncer gástrico difuso
}

\author{
Blumer's shelf: diagnostic sign of distant spread of diffuse gastric cancer
}

\author{
Aingeru Sarriugarte-Lasarte ${ }^{\mathbb{D}}$, Eva García-Alberdi², Oihane Gutierrez-Grijalba ${ }^{3}$, \\ Patricia Mifsut-Porcel ${ }^{1}$, Laura Fernández Gómez-Cruzado ${ }^{3}$ D
}

\footnotetext{
1 Médico, especialista en Cirugía general y Aparato digestivo, Hospital universitario de Cruces, Biocruces. EHU/UPV. Barakaldo, Vizcaya, España.

2 Médico, especialista en Anatomía patológica, Hospital universitario de Cruces, Barakaldo, Vizcaya, España.

3 Médico, especialista en Cirugía general y Aparato digestivo, Hospital universitario de Cruces, Barakaldo, Vizcaya, España.
}

Trabajo presentado como poster en la XXVI Reunión Nacional de Cirugía Esófago Gástrica ISDE 2019 en Bilbao.

\section{Resumen}

Introducción. Se conoce como escudo de Blumer al engrosamiento neoplásico del fondo de saco de Douglas, palpable al tacto rectal como una protrusión en forma de resalte, presente en pacientes afectados por carcinomas con infiltración difusa. A pesar de haber sido descrito hace más de un siglo, se trata de un proceso diagnosticado infrecuentemente y con escasa mención en la literatura científica. Es preciso tener un alto nivel de sospecha clínica para correlacionar los síntomas pélvicos con la presencia de un tumor, habitualmente gástrico.

Casos clínicos. Presentamos dos pacientes con hallazgo de escudo de Blumer, asociado a cáncer gástrico difuso, uno como diagnóstico primario de enfermedad metastásica y otro como recidiva de la enfermedad, meses después de ser operado. Ambos casos presentan un complejo proceso diagnóstico, en el que prima la sospecha clínica, apoyado sobre pruebas de imagen como tomografía computarizada y resonancia nuclear magnética, ya que tanto las biopsias obtenidas por endoscopia, como las biopsias intraoperatorias fueron negativas.

Discusión. En el escudo de Blumer, las células tumorales infiltran el fondo de saco de Douglas de forma difusa por debajo de la serosa, sin necesidad de que existan implantes macroscópicamente visibles en el peritoneo visceral. La infiltración tumoral puede afectar extrínsecamente al recto, causando una estenosis del mismo, lo que produce sintomatología pélvica inespecífica, como tenesmo rectal y proctalgia. Por lo tanto, este ominoso proceso debe ser sospechado en pacientes con sintomatología pélvica, que presenten o hayan presentado cáncer gástrico.

Palabras clave: estómago; neoplasias gástricas; adenocarcinoma; linitis plástica; escudo de Blumer.

Fecha de recibido: 07/01/2020 - Fecha de aceptación: 08/05/2020

Correspondencia: Aingeru Sarriugarte-Lasarte, Olalde Beresia 4- 4B 48100 Mungia. Bizkaia. España. Teléfono: +34 635209305

Correo electrónico: aingeru.sarriugartelasarte@osakidetza.eus

Citar como: Sarriugarte-Lasarte A, García-Alberdi E, Gutierrez-Grijalba O, Mifsut-Porcel P, Fernández Gómez-Cruzado L. Escudo de Blumer: signo diagnóstico de la diseminación a distancia del cáncer gástrico difuso. Rev Colomb Cir. 2021;36:144-9. https://doi.org/10.30944/20117582.547

Este es un artículo de acceso abierto bajo una Licencia Creative Commons - BY-NC-ND https://creativecommons.org/licenses/by-ncnd/4.0/deed.es 


\begin{abstract}
Introduction. The neoplastic thickening of the cul-de-sac of Douglas is known as "Blumer's shelf". It is palpable on rectal examination as a protrusion in the form of a projection, and it presents in patients affected by carcinomas with diffuse infiltration. Despite being described more than a century ago, it is a rare process with little mention in the scientific literature. A high level of clinical suspicion is required to correlate pelvic symptoms with the presence of a typically gastric tumor.

Clinical cases. We present two patients with a Blumer's shelf finding associated with diffuse gastric cancer, one as a primary diagnosis of metastatic disease and the other as a recurrence of the disease, months after being operated on. Both cases present a complex diagnostic process, in which clinical suspicion prevails, supported by imaging tests such as computed tomography and magnetic resonance imaging, since both endoscopic biopsies and intraoperative biopsies were negative.

Discussion. In Blumer's shelf, tumor cells infiltrate the cul-de-sac of Douglas in a diffuse and subserous manner, without the need for macroscopically visible implants in the visceral peritoneum. Tumor infiltration can extrinsically affect the rectum, causing its stenosis, which produces nonspecific pelvic symptoms such as rectal tenesmus and proctalgia. Therefore, this ominous process should be suspected in patients with pelvic symptoms, who present or have presented gastric cancer.
\end{abstract}

Keywords: stomach; gastric neoplasms; adenocarcinoma; plastic linitis; Blumer shelf.

\section{Introducción}

Se conoce como escudo de Blumer al engrosamiento neoplásico del fondo de saco de Douglas, palpable al tacto rectal que, excepcionalmente se presenta como manifestación a distancia en pacientes afectados por carcinoma con infiltración difusa. A pesar de haber sido descrito hace más de un siglo, no existe prácticamente literatura que correlacione este ominoso signo con las pruebas de imágenes y la anatomía patológica. Presentamos dos casos de pacientes con adenocarcinoma gástrico difuso, en los cuales la presencia del escudo de Blumer auguró la diseminación a distancia de la enfermedad, que solo se pudo confirmar mediante cirugía y anatomía patológica.

\section{Caso 1}

Mujer de 80 años, con estudios previos por diarrea y tenesmo rectal, al tacto rectal se encontró un engrosamiento rectal en la cara anterior, sin irregularidad mucosa. Se estudió mediante tomografía computarizada (TC) (figura 1a y 1b), con hallazgo de engrosamiento rectal ya sospechado y neoplasia gástrica, y colonoscopia donde se encontró una estenosis rectal extrínseca (figura 1c), secundaria al engrosamiento de recto que imposibilita el paso del endoscopio, sin hallazgos anatomopatológicos de malignidad sobre la mucosa. La gastroscopia encontró un adenocarcinoma gástrico pobremente diferenciado T3N2.

Se realizó laparoscopia exploratoria, hallando linitis plástica del estómago, sin datos de carcinomatosis peritoneal macroscópica. Se encontró engrosamiento preperitoneal del fondo de saco de Douglas (figura 1d y 1e), con compromiso subseroso por la cara anterior del recto, causando estenosis rectal secundaria. Se realizaron múltiples biopsias intraoperatorias peritoneales y de peritoneo pélvico, siendo todas negativas para malignidad. A pesar de sospechar la posibilidad de enfermedad diseminada, ante la ausencia de claras imágenes de implantes peritoneales, y de biopsias que confirmaran la carcinomatosis peritoneal, se realizó gastrectomía total abierta, linfadenectomía D2 y colostomía del sigmoide en la fosa iliaca izquierda.

La paciente presentó un posoperatorio favorable, siendo dada de alta al octavo día. La anatomía patológica definitiva informó adenocarcinoma gástrico difuso T4aN3a y M1 por positividad para 


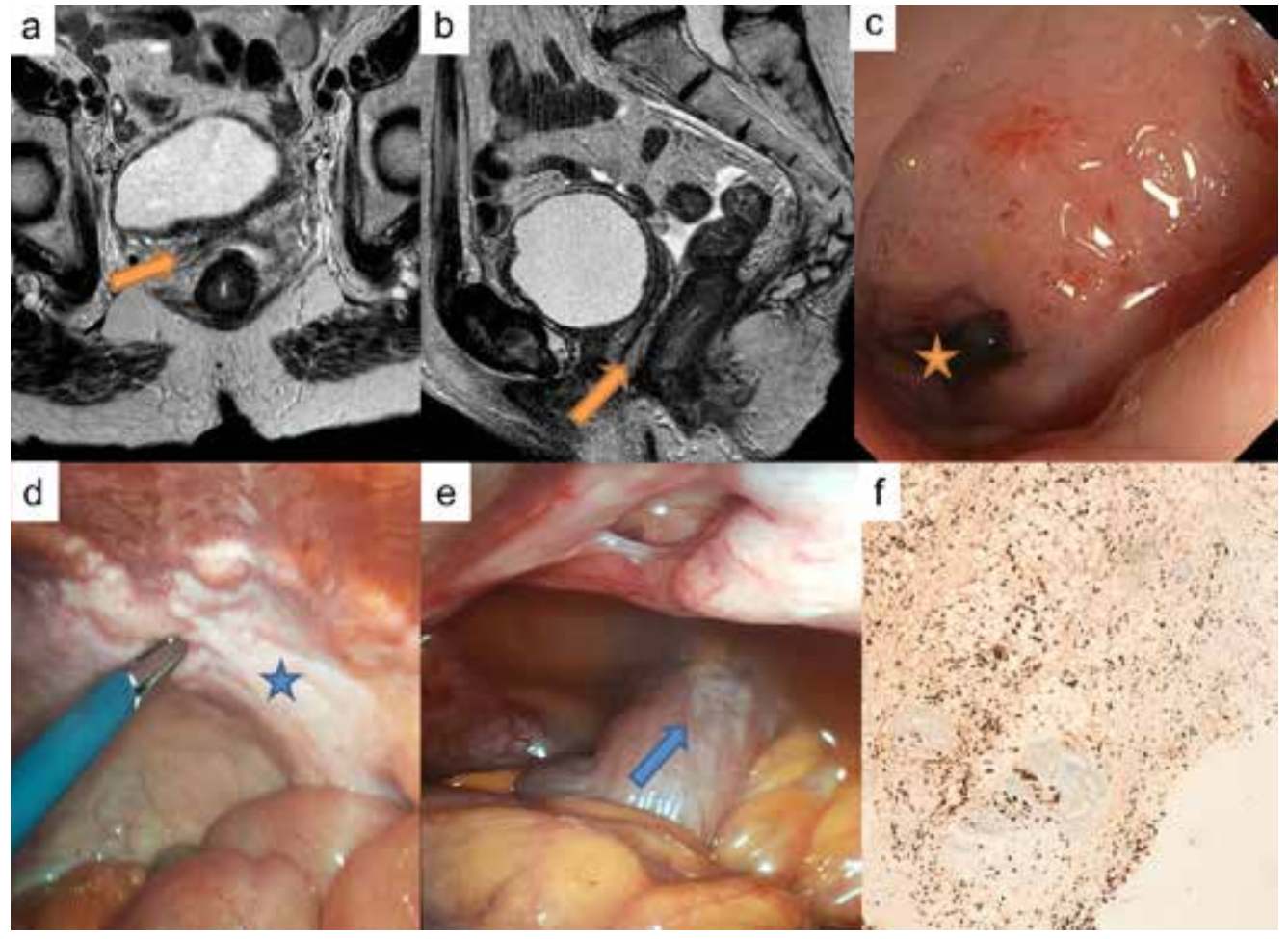

Figura 1. Caso 1

a, b) Imagen axial y sagital de tomografía computarizada, donde se aprecia el engrosamiento rectal extrínseco y del saco de Douglas (flecha naranja). c) Imagen endoscópia de la estenosis extrínseca (estrella naranja) con mucosa de aspecto normal. d) Imagen de laparoscopia donde se aprecia el engrosamiento del saco de Douglas o "escudo de Blumer" (estrella azul). e) Imagen laparoscópica donde se aprecia el compromiso tumoral rectal subseroso o linitis plástica rectal secundaria (flecha azul). f) Imagen microscópica de anatomía patológica de la biopsia del saco de Douglas con infiltración subserosa por carcinoma difuso. Inmunohistoquímica de CK8+, aumento x10

afectación por carcinoma difuso encontrado en las biopsias del peritoneo de fondo de saco de Douglas con compromiso subseroso (figura 1f). La paciente vivió 12 meses con aceptable tolerancia oral y tránsito intestinal conservado, presentó recidiva peritoneal al octavo mes, sin recibir tratamiento adyuvante debido a su edad y comorbilidades.

\section{Caso 2}

Paciente masculino de 67 años, tratado por adenocarcinoma gástrico difuso T3N1 mediante quimioterapia perioperatoria con esquema EOX, gastrectomía total y linfadenectomía D2. Anatomía patológica con bordes libres y citología peritoneal negativa. El seguimiento mediante TC y gastroscopia cada 6 meses, resultó normal hasta el décimo sexto mes, cuando presentó tenesmo rectal y elevación de los marcadores tumorales. Al tacto rectal se encontró rugosidad en la cara anterior rectal, no dolorosa y de aspecto extrínseco. Se realizó TC y resonancia magnética nuclear (RMN), (figuras 2a y 2b), observando el engrosamiento circunferencial de las paredes del recto y del sigmoide distal, de aspecto congestivo, sin visualizar lesiones neoplásicas primarias de recto.

Se completó el estudio mediante colonoscopia y ultrasonografía endoscópica, encontrando un discreto engrosamiento de la submucosa e hiperecogenicidad a nivel de la grasa del meso rectal. Todas las biopsias endoscópicas fueron negativas, 
y se observó predominio del tejido conectivo, con ausencia de celularidad. Se realizó laparoscopia exploratoria, encontrando el engrosamiento del fondo del saco peritoneal, conocido como escudo de Blumer (figura 2c), sin hallazgos claros de carcinomatosis peritoneal macroscópica. Las biopsias quirúrgicas resultaron positivas para infiltración subserosa por carcinoma difuso (figura $2 \mathrm{~d}, 2 \mathrm{e} y$ 2f) por lo que se realizó tratamiento paliativo mediante quimioterapia.

\section{Discusión}

El escudo de Blumer, también conocido como anaquel de Blumer, fue descrito en 1909 por el ciruja- no ingles George Blumer, como un engrosamiento palpable al tacto rectal, signo de mal pronóstico, producido por la diseminación a distancia de procesos neoplásicos o inflamatorios desconocidos ${ }^{1}$. Este signo ha sido olvidado o abandonado, por lo que se hace hincapié en que el tacto rectal debe continuar siendo parte primordial de la exploración física, en los pacientes oncológicos.

A pesar de haber sido descrito hace más de un siglo y de tratarse, al igual que el nódulo de la hermana María José (nódulo umbilical metastásico) o el ganglio de Virchow (ganglio supraclavicular izquierdo), de un signo clásico de diseminación del cáncer gástrico, prácticamente no existe lite-

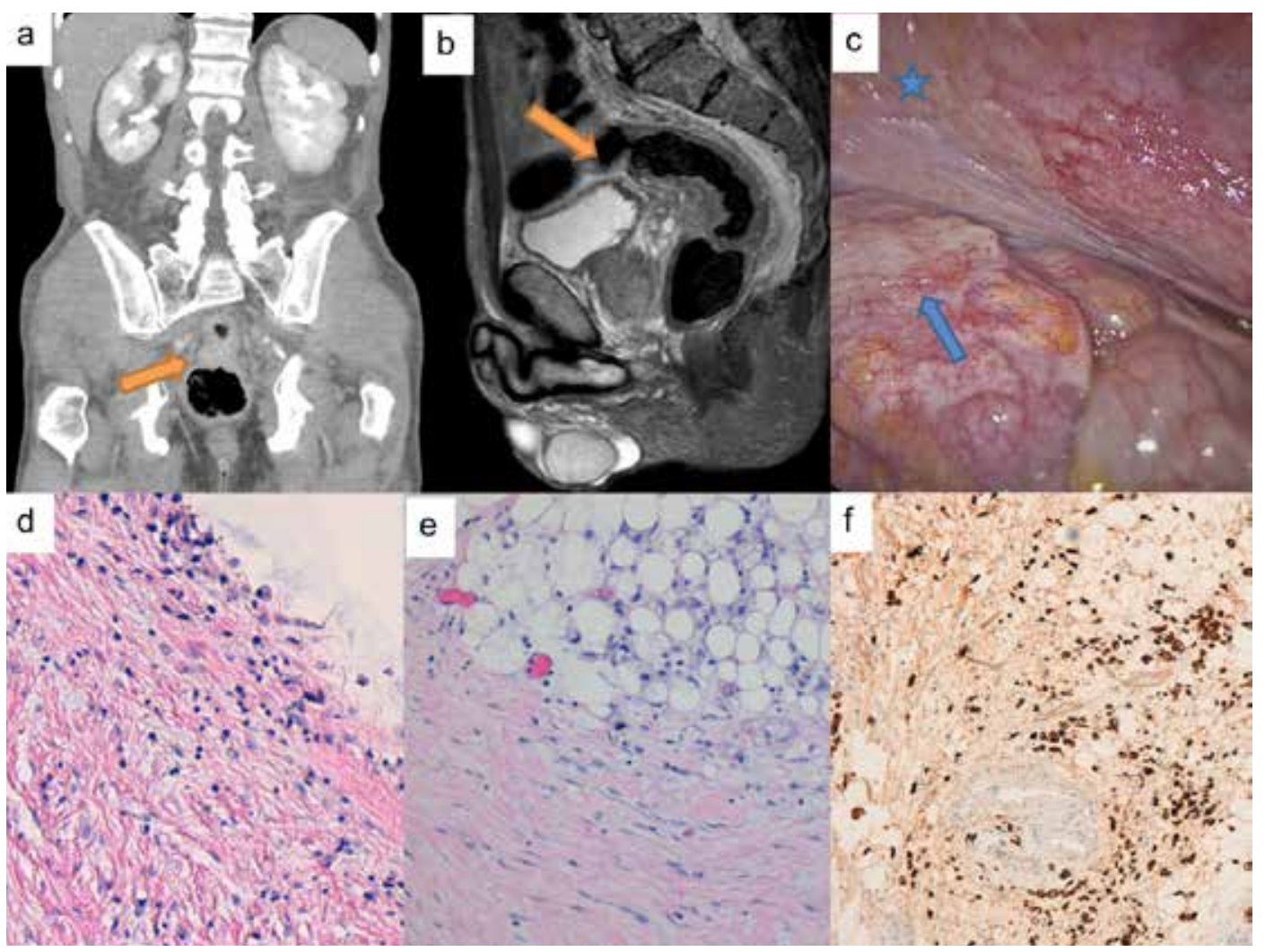

Figura 2. Caso 2

a, b) Imagen axial y sagital de resonancia nuclear, donde se aprecia el engrosamiento rectal extrínseco y del saco de Douglas (flecha naranja). c) Imagen de laparoscopia, donde se aprecia el engrosamiento del saco de Douglas o "escudo de Blumer" (estrella azul) y el compromiso tumoral rectal subseroso o linitis plástica rectal secundaria (flecha azul). d, e) Imágenes microscópicas de anatomía patológica, de las biopsias del saco de Douglas con infiltración subserosa por carcinoma difuso, HE (Hematoxilina Eosina) aumento x10.f) Imágenes microscópicas de anatomía patológica, de las biopsias del saco de Douglas con infiltración subserosa por carcinoma difuso. Inmunohistoquímica de CK8+, aumento x10. 
ratura que correlacione la clínica, la exploración y los estudios imagenológicos, con los hallazgos intraoperatorios y de anatomía patológica de este funesto proceso ${ }^{2,3}$.

En estos casos, la diseminación peritoneal de la enfermedad se produce de manera característica. Sin necesidad de que existan implantes macroscópicamente visibles en el peritoneo visceral intrabdominal, las células tumorales infiltran el fondo de saco de Douglas, el cual se observa engrosado y con claro aspecto patológico, pero sin compromiso de la serosa peritoneal o aspecto nodular. Microscópicamente se observa aumento del tejido conectivo en la capa subserosa, conformado por estroma maduro e inmaduro, con escasa celularidad, en algunas ocasiones en forma de anillo de sello. La infiltración tumoral puede afectar extrínsecamente al recto, convirtiéndolo en un tubo rígido, pero manteniendo la mucosa intacta, en lo que se conoce como linitis plástica rectal secundaria ${ }^{4}$ que aunque habitualmente hace referencia a la diseminación del cáncer gástrico difuso, excepcionalmente puede presentarse como metástasis de tumores de vejiga, mama o pulmón ${ }^{5}$.

El escudo de Blúmer se manifiesta en estadios tardíos de la enfermedad, tanto como diagnóstico primario con afectación peritoneal, como muestra el primer caso, o bien como recidiva peritoneal a distancia durante el seguimiento, como muestra el segundo caso. Suele presentarse de manera silente, produciendo síntomas vagos como el estreñimiento, tenesmo rectal o molestias pélvicas. Un alto nivel de sospecha y el tacto rectal, que debe seguir siendo una parte importante de la exploración física de estos pacientes, son la primera llamada de atención, que sugiere una neoplasia abdominal diseminada ${ }^{6}$.

No obstante, en la actualidad, pruebas de imagen como la endoscopia, la TC y la RMN pélvicas, son de gran ayuda en el diagnóstico de la neoplasia primaria, de la confirmación de la afectación neoplásica del tabique rectal, o del diagnóstico diferencial con lesiones inflamatorias pélvicas. En la endoscopia se encuentra una pared rectal engro- sada, con una mucosa de aspecto normal, lo que dificulta el diagnóstico. La escasa celularidad hace que hasta en la mitad de los casos, las muestras de anatomía patológica sean negativa para malignidad $^{6}$. La TC, y más concretamente la RMN pélvica, son útiles aunque presentan un importante número de falsos negativos. Estudios recientes mediante endosonografía muestran su gran utilidad, ya que es capaz de valorar con alta sensibilidad la afectación de la submucosa muscular propia y subserosa, permitiendo la obtención dirigida de material para estudio histológico. No obstante, ante la duda diagnóstica y la imposibilidad de determinar el origen del engrosamiento, en algunos casos es necesaria la laparoscopia exploratoria, con el fin de obtener biopsias quirúrgicas ${ }^{7}$.

\section{Conclusiones}

El escudo de Blumer es el engrosamiento del fondo de saco de Douglas, palpable al tacto rectal, que ocurre como consecuencia de la diseminación a distancia del carcinoma gástrico. Se presenta en pacientes con sintomatología pélvica, o con síntomas vagos, y debe hacer sospechar la presencia de cáncer gástrico, o su relación con el antecedente del mismo.

\section{Cumplimiento de normas éticas}

Consentimiento informado: Los autores confirman tener el consentimiento de los pacientes para la publicación del caso.

Conflictos de interés: Los autores declaran no tener conflictos de interés.

Financiación: No se han recibido ningún apoyo para la realización del estudio.

Contribuciones de los autores: Concepción y diseño del estudio, adquisición de datos, análisis e interpretación de datos: Aingeru Sarriugarte-Lasarte, Patricia Mifsut-Porcel. Redacción del manuscrito y revisión crítica: Eva GarcíaAlberdi, Oihane Gutiérrez-Grijalba, Laura Fernández Gómez-Cruzado.

Los autores declaramos haber revisado y convalidado el contenido del manuscrito 


\section{Referencias}

1. Blumer G. Rectal shelf: neglected rectal sign of value in diagnosis of obscure malignant and inflammatory disease within the abdomen. Albany Medical Annals. 1909;30:361.

2. Ahmad SA, Xia BT, Bailey CE, Abbott DE, Helmink BA, Daly MC, et al. An update on gastric cancer. Current Problems in Surgery. 2016;53:449-90. https://doi.org/10.1067/j.cpsurg.2016.08.001

3. Clark CJ, Thirlby RC, Picozzi V, Schembre DB, Cummings FP, Lin E. Current Problems in Surgery: Gastric Cancer. Curr Probl Surg. 2006;43:566-670.

https://doi.org/10.1067/j.cpsurg.2006.06.003

4. Katsinelos P, Papaziogas B, Chatzimavroudis G, Katsinelos T, Dimou E, Atmatzidis S. Secondary rectal linitis plastica as first manifestation of urinary bladder carcinoma. Ann Gastroenterol. 2012;25:175-5.

5. Rodríguez-Ortega M, Carabias-Hernández A, Rodríguez-Barbero JM, Montano-Navarro E, Limones-Esteban M. Linitis plástica intestinal, metástasis tardía de adenocarcinoma gástrico en anillo de sello. Rev Esp Enferm Dig. 1998;5:391-2.

6. Küttner-Magalhães R, Mesquita I, Brandão PN, Peixoto C, Varzin P, Rodríguez A. Endoscopic appearance of Blumer's shelf. Gastrointest Endosc. 2015;82(2):412-413. https://doi.org/10.1016/j.gie.2015.02.011

7. Gómez-Moreno AZ. Hallazgos endoscópicos y ecoendoscópicos en la linitis plástica rectal secundaria, Gastroenterol Hepatol. 2011;34:535-8. 